BMC

Genomics

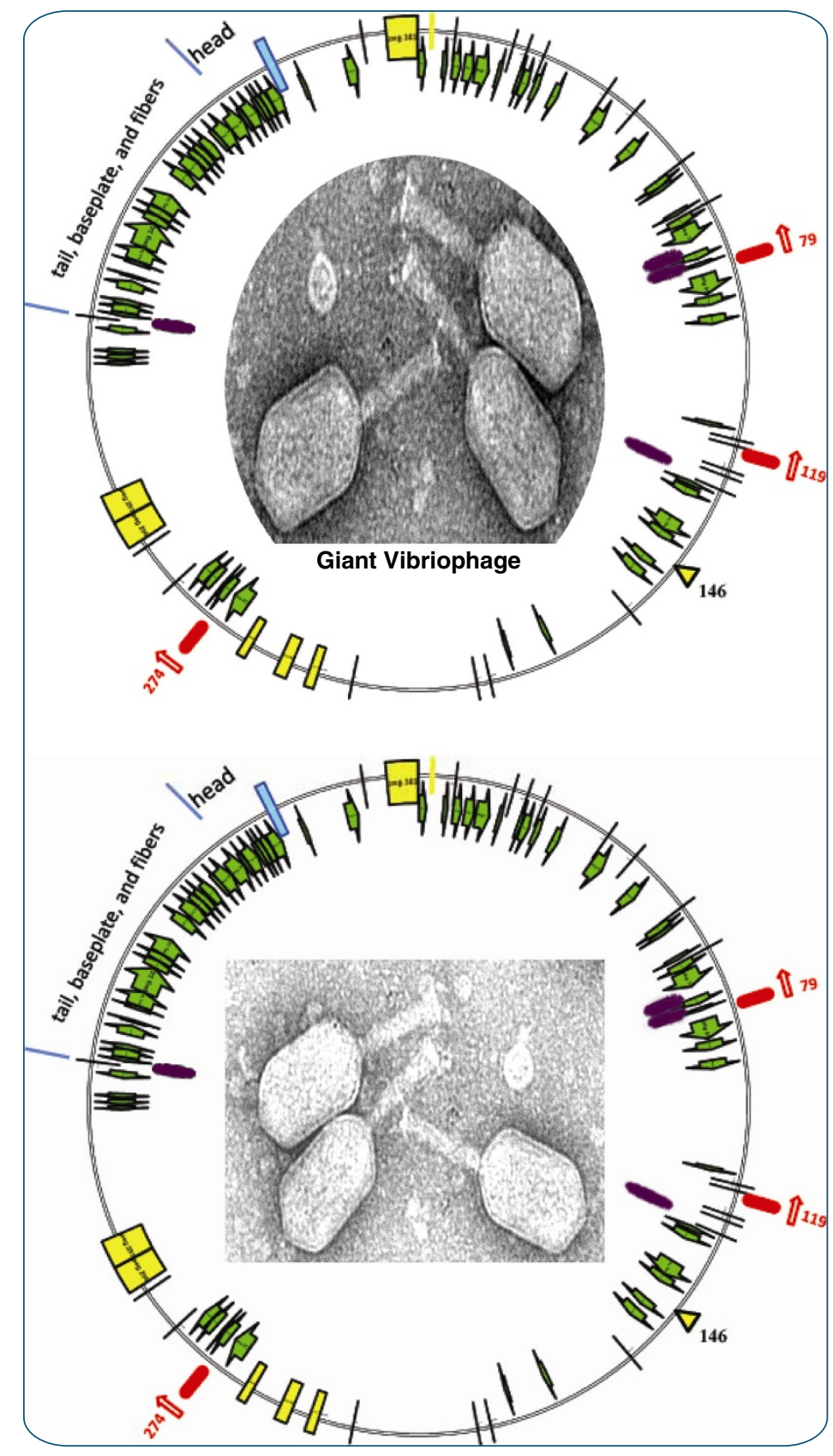

Genome-wide characterization of vibrio phage بpp2 with unique arrangements of the mob-like genes

Lin and Lin

C Biomed Central

Lin and Lin BMC Genomics 2012, 13:224

http://www.biomedcentral.com/1471-2164/13/224 


\title{
Genome-wide characterization of vibrio phage بpp2 with unique arrangements of the mob-like genes
}

Ying-Rong Lin ${ }^{1}$ and Chan-Shing Lin ${ }^{1,2^{*}}$

\begin{abstract}
Background: Vibrio parahaemolyticus is associated with gastroenteritis, wound infections, and septicemia in human and animals. Phages can control the population of the pathogen. So far, the only one reported genome among giant vibriophages is KVP40: 244,835 bp with 26\% coding regions that have T4 homologs. Putative homing endonucleases (HE) were found in Vibrio phage KVP40 bearing one segD and Vibrio cholerae phage ICP1 carrying one $\mathrm{mobC/E}$ and one segG.

Results: A newly isolated Vibrio phage $\varphi p p 2$, which was specific to the hosts of $\mathrm{V}$. parahaemolyticus and $\mathrm{V}$. alginolyticus, featured a long nonenveloped head of $\sim 90 \times 150 \mathrm{~nm}$ and tail of $\sim 110 \mathrm{~nm}$. The phage can survive at $50^{\circ} \mathrm{C}$ for more than one hour. The genome of the phage $\varphi p p 2$ was sequenced to be $246,421 \mathrm{bp}$, which is $1587 \mathrm{bp}$ larger than KVP40. 383 protein-encoding genes (PEGs) and 30 tRNAs were found in the phage $\varphi p p 2$. Between the genomes of $\varphi$ pp2 and KVP40, 254 genes including 29 PEGs for viral structure were of high similarity, whereas 17 PEGs of KVP40 and 21 PEGs of $\varphi p p 2$ were unmatched. In both genomes, the capsid and tail genes have been identified, as well as the extensive representation of the DNA replication, recombination, and repair enzymes. In addition to the three giant indels of 1098, 1143 and $3330 \mathrm{nt}$, $\varphi p p 2$ possessed unique proteins involved in potassium channel, gp2 (DNA end protector), tRNA nucleotidyltransferase, and mob-type HEs, which were not reported in KVP40. The $\varphi p p 2$ PEG274, with strong promoters and translational initiation, was identified to be a mobE type, flanked by NrdA and NrdB/C homologs. Coincidently, several pairs of HE-flanking homologs with empty center were found in the phages of Vibrio phages Ypp2 and KVP40, as well as in Aeromonas phages (Aeh1 and Ae65), and cyanophage P-SSM2.

Conclusions: Vibrio phage $\varphi p p 2$ was characterized by morphology, growth, and genomics with three giant indels and different types of HEs. The gene analysis on the required elements for transcription and translation suggested that the ppp2 PEG274 was an active mobE gene. The phage was signified to be a new species of T4-related, differing from KVP40.
\end{abstract}

Keywords: Homing endonuclease, T4-like phage, Vibrio parahaemolyticus

\section{Background}

Vibrio parahaemolyticus is a halophilic gram-negative bacterium that is widely distributed in coastal waters worldwide and is associated with gastroenteritis, wound infections, and septicemia [1]. Since the first report of Fujino et al. [2], numerous investigations of

\footnotetext{
* Correspondence: shinlin@faculty.nsysu.edu.tw

'Department of Marine Biotechnology and Resources, Asia-Pacific Ocean Research Center, National Sun Yat-sen University, Kaohsiung 80424, Taiwan ${ }^{2}$ Agricultural Biotechnology Center, National Chung-Hsing University, Taichung 402, Taiwan
}

$V$. parahaemolyticus have been performed using stools of patients and diseased fish. The halophile has been found seasonally in sea water of the continental United States, Germany, the Far East, and Hawaii [3-6]. V. parahaemolyticus infections are frequently reported to occur due to the consumption of undercooked raw shellfish or direct contact with estuarine waters. In Asia, many recent infections have been caused by serotype O3:K6 of $V$. parahaemolyticus [7].

The phages can control the population of the pathogen. Among the giant T4-like phages that are specific to

\section{Biomed Central}


$V$. parahaemolyticus, the vibriophage KVP40 is the only strain for which the genome has been determined [8]. The size of the KVP40 genome is 244,835 bp with an overall $\mathrm{G}+\mathrm{C}$ content of $42.6 \%$. It contains 381 putative protein-encoding genes (PEG), 30 tRNAs, 33 late promoters, and 57 rho-independent terminators. The genome sequence and organization of KVP40 show a degree of conservation with phage T4. While $65 \%$ of the PEGs were unique to KVP40, 99 out of the total 381 putative coding regions have homologs in the $\mathrm{T} 4$ genome, which includes DNA replication, recombination, and repair enzymes as well as the viral capsid and tail structural genes. KVP40 lacks enzymes involved in DNA degradation, cytosine modification and group I introns, and it probably utilizes NAD salvage pathway that is unique among bacteriophages [8].

Phages can prompt gene recombination via homing endonucleases (HEs). In genome analyses, putative homing endonucleases (HEs) were found in Vibrio parahaemolyticus phages KVP40 and Vibrio cholerae ICP1 [8,9]. Homing endonucleases might act as possible mediators for the diversity among bacteriophage genomes by the acquisition of a novel DNA to create a new species of phage. Although more than 30 T4-related genomes have been published so far, no other known phage genome comes close to encoding the 15 homing endonucleases in T4 phage [10-12]. Intron homing [13] and intronless homing $[14,15]$ endonucleases both utilize homologous recombination between phages to transfer the genetic elements from the HE-encoding genome to a HElacking recipient. The seg and $m o b$ subtypes, which are also called freestanding endonucleases, belong to the GIY-YIG and HNH homing endonuclease families, respectively [16, a review]. The segC, segF, segG, mobA, and $m o b \mathrm{E}$ of $\mathrm{T} 4$ endonucleases are polycistronically transcribed with their respective upstream genes, whereas the endonuclease-specific promoters for segA, segG, mobC and mobD are immediately upstream of the endonuclease genes [16]. There is as yet no convincing evidence that the HEs can move across the boundary of species or genera. Nevertheless, these transposable genes may leave a trace of their involvement after the transfers. The sequence analysis for the Enterobacteria phage JSE intron revealed that the putative intron contained a truncated derivative of a HE gene [17], very similar to the truncated sequence in the intron of the $\mathrm{T} 4 \mathrm{nrdB}$ gene, suggesting that there is a rarely-detectable trace of the $\mathrm{mob} / \mathrm{seg}$ elements in contemporary phage genomes [18].

We sequenced the genome of $\phi p p 2$ - a new T4-like Vibrio phage with mob genes - which may be another paradigm in the plausible analysis of evolution of $\mathrm{HE}$ families in the bacteriophages and their hosts [8]. In the same host, Vibrio parahaemolyticus, the phage $\phi p p 2$ can complement KVP40 in studying the genome spectra of the giant T4-related Vibrio phages.

\section{Methods}

\section{Bacteria strains and growth conditions}

Vibrio strains were bought from the Bioresource Collection and Research Center, Taiwan; including $V$. alginolyticus ATCC 17749, V. carchariae ATCC 35084, V. damsela ATCC 33536, V. harveyi ATCC 14126, V. parahaemolyticus ATCC 17802, V. pelagius ATCC 25916, and $V$. vulnificus BCRC15431. V. parahaemolyticus ATCC 17802 carries O1 serotype and no $t d h / t r h$ genes [19]. The Vibrio strains were maintained in Brain Heart Infusion (BHI) medium, supplemented with $3 \% \mathrm{NaCl}$. For long-term preservation, bacteria were frozen in BHI supplemented with $1 \% \mathrm{NaCl}$ and $25 \%$ glycerol. When working, the strains were streaked onto the modified sea water yeast extract (rich MSWYE) agar plates consisting of $23.4 \mathrm{~g} \mathrm{NaCl}, 6.98 \mathrm{~g} \mathrm{MgSO} 47 \mathrm{H}_{2} \mathrm{O}$, and $0.75 \mathrm{~g} \mathrm{KCl}$ in $1000 \mathrm{ml}$ distilled water [19]. The $\mathrm{pH}$ was adjusted to 7.6 with $1 \mathrm{~N} \mathrm{NaOH}$, followed by addition of $5.0 \mathrm{~g}$ of proteose peptone (Difco), $3.0 \mathrm{~g}$ of yeast extract (Difco), and $20.0 \mathrm{~g}$ of agar per liter.

\section{Isolation and titer of bacteriophage}

The water samples were collected from the aquaculture waterways around southern Taiwan. The enrichment procedure for the target phages has been described elsewhere [20]. In brief, 20\% of MSWYE medium and 1\% seed culture of Vibrio parahaemolyticus were added the micro-filtrated samples and incubated at $37^{\circ} \mathrm{C}$ for four hours to enrich the phages. In determining the phage concentrations, the bacterium Vibrio parahaemolyticus was freshly grown to $0.3-0.4 \mathrm{OD}_{600}$, in about two hours, and $200 \mu \mathrm{l}$ of cells were added to $10 \mu \mathrm{l}$ phages in a series of dilutions for infection, followed by the Agar Overlay Technique. The plaques were counted in 3-5 hours; the titers per $\mathrm{ml}$ were calculated by $100 \%$ (dilution factor)* (plaque counts).

\section{Electron microscopy}

Preparation of phage particles for electron microscopy has been described elsewhere [20,21]. In brief, bacteriophage particles were applied onto parafilm to produce a spherical drop. Carbon-coated nitrocellulose films were fabricated on copper grids and placed face down on the sample drops for $1 \mathrm{~min}$ to absorb the particles. The samples were stained with freshly prepared $2 \%$ uranyl acetate (UA; Tris- $\mathrm{HCl}, \mathrm{pH} 8.0$ ) for 60 seconds. Images of phage particles were taken at a magnification of 40,000x, defocus of $3 \mu \mathrm{m}$, using a 200-kV electron microscope (JEOL JEM-2010, equipped with a Gatan-832 CCD camera). 


\section{Analyses of bacteriophage DNA}

In phage propagation, ten milliliters of $\phi \mathrm{pp} 2$ phage stock were added to $50 \mathrm{ml}$ of $V$. parahaemolyticus (3x $10^{8}$ CFU ml ${ }^{-1}$ ) cultured in MSWYE, incubated in a shaker at $37^{\circ} \mathrm{C}$ for $3-5$ hours, when the lysate was clear with some cell debris. The remaining cells and debris were removed by two centrifugations at $10000 \times g$ for $30 \mathrm{~min}$ utes. With an optimal titer of $4 \times 10^{9} \mathrm{PFU} \mathrm{ml}{ }^{-1}$, the supernatant was stored at $4^{\circ} \mathrm{C}$ as a phage stock. To concentrate phages using a standard protocol with polyethylene glycol precipitation [22-24], solid $\mathrm{NaCl}(0.6 \mathrm{M})$ and polyethylene glycol 8000 (20\%) were added and precipitation was performed overnight at $4^{\circ} \mathrm{C}$. After centrifugation, the phage particles were resuspended in $2 \mathrm{ml}$ of SM buffer and treated with DNase I and RNase A to remove contamination of host nucleotides. The polyethylene glycol was extracted by adding an equal volume of chloroform until the interface was clear. The aqueous phase containing phages was treated with Proteinase $\mathrm{K}$ and sodium dodecyl sulfate (SDS) at $56^{\circ} \mathrm{C}$ for $1 \mathrm{~h}$. Phenol extraction was carried out three times at room temperature; the aqueous phase was further extracted with a 1:1 mixture of equilibrated phenol and chloroform. DNA precipitated by $2 \times$ volume of cold ethanol was re-dissolved in deionized water.

\section{Thermal stability of phage $\varphi p p 2$}

Thermal stability tests have been described elsewhere $[25,26]$. Briefly, the bacterium Vibrio parahaemolyticus was freshly inoculated at the $1 \%$ volume of seed from overnight culture into $20 \mathrm{ml}$ of rich MSWYE broth. When the cell density reached $0.4-0.5 \mathrm{OD}_{600}$, the treated phages of a series dilution were added to infect the host for 5 minutes, mixed with top agar, and poured onto a solid surface of regular agar plate in order to count the plaques in 3-5 hours. $2 \times 10^{9} \mathrm{PFU}$ of phage particles were treated under $37-80^{\circ} \mathrm{C}$ and samples were taken at 15 -min intervals. The supernatants from the centrifugation of $14000 \times g$ for 3 minutes were diluted and titered for phage numbers by Agar-overlay method.

\section{Genome sequencing and annotation}

Similar to shotgun sequencing described elsewhere, approximately $5 \mu \mathrm{g}$ of the bacteriophage genomic DNA was randomly sheared by nebulization, and DNA sequencing was performed at Mission Biotech according to the manufacturer's protocol for the Genome Sequencer GS Junior System (Roche Diagnostic). Low quality sequences of the reads generated by the GS Junior sequencer were trimmed off. De novo assembly of the shotgun reads was performed with the GS Assembler software. Sequence assembly and analyses were performed essentially as described previously [27]. Proteincoding genes (PEG) were predicted using The RAST
Server (Rapid Annotations using Subsystems Technology; http://rast.nmpdr.org/) [28] and analyzed with the SEED- Viewer (http://www.theseed.org/wiki/Main_Page) [29]. Protein-coding genes were also checked using the $a b$ initio gene-finding program Glimmer v3.02 [30]. rRNA genes of the draft assembly were identified using RNAmmer [31]. tRNA genes for all 20 amino acids that were predicted by the RAST were further verified using tRNAscan-SE [32]. Automatic functional annotation results obtained by the RAST were further compared with the proteins in the GenBank database using PSIBLAST (www.ncbi.nlm.nih.gov/blast/Blast.cgi). The Neural Network Promoter Prediction (NNPP) program was used to find the promoters [33].

\section{Multiple sequence alignments}

To determine the taxonomy status of the new phage isolate $\phi \mathrm{pp} 2$, the genome sequence data of Enterobacteria phage T4 and Vibrio phages KVP40 were employed to find the high homologous regions with the new phage after PSI-BLAST searches. Complete genome sequences of the Vibrio T4-like phages were acquired from NCBI, including Enterobacteria phage T4 (168903 bp in GenBank access no. NC_000866), Vibrio phage KVP40 (244834 bp in GenBank access no. NC_005083), Aeromonas phage 65 (235229 bp in GenBank access no. NC_015251), Aeromonas phage Aeh1 (233234 bp in GenBank access no. NC_005260), and Prochlorococcus phage P-SSM2 (252401 bp in GenBank access no. NC_006883). T4-like myoviruses also include Enterobacteria phages RB14 (NC_012638), RB16 (NC_014467), RB32 (NC_008515), RB51 (NC_012635), JS10 (NC_012741), and JSE (NC_012740), Aeromonas phages 325(NC_008208), and Vibrio cholerae phage ICP1 (NC_015157). PBCV-1 is the Paramecium bursaria Chlorella virus 1. Sequences of individual target genes retrieved from the genome sets were then aligned using ClustalW with default options [34]. The best alignments of individual genes were analyzed by a neighbor-joining method using the NEIGHBOR program in Phylogeny Inference Package (PHYLIP) [35]. Distances were calculated using the PROTDIST programs of PHYLIP and displayed in TreeView [36]. The ClustalW, PHYLIP, and TreeView were bundled in the BioEdit program version 7.0 .5 [37].

\section{Results}

\section{Phage morphology}

The morphology of phage $\phi \mathrm{pp} 2$ was observed by transmission electron microscopy, which is traditionally one of the most frequently used methods to classify phages. As Figure 1 shows, $\phi p p 2$ was a large phage with nonenveloped head, neck, collar, and tail: the head was approximately $90-95 \mathrm{~nm}$ wide by $150-160 \mathrm{~nm}$ long and the tail was about $110-120 \mathrm{~nm}$ long with $20-25 \mathrm{~nm}$ in diameter. 


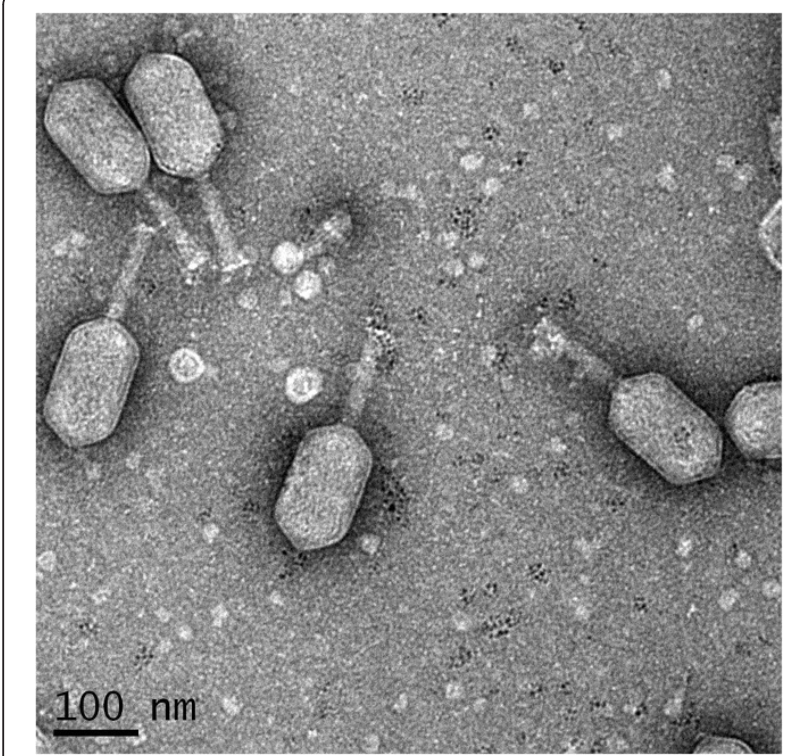

Figure 1 Transmission electron micrograph of phage $\varphi p p 2$ particles with several structural proteins. The phage particles were purified with three times of centrifugations by PEG-NaCl precipitation method mentioned in the Material section. Virion particles were negatively stained with uranyl acetate for EM. The bars represent a length of $100 \mathrm{~nm}$.

A baseplate and tail pins were observed under different focus, while long tail-fibers were threading randomly.

\section{Host range}

The susceptibility of seven Vibrio strains to the phage фpp2 was also investigated with the Agar-overlay method. Among them, $V$. parahaemolyticus, $V$. damsela and $V$. alginolyticus were found susceptible to phage фpp2 while the other four species ( $V$. carchariae, $V$. damsela, $V$. harveyi, $V$. pelagius, and $V$. vulnificus) could not be infected even at high MOI.

\section{Viability of phage $\varphi p p 2$ in the thermal environment}

Thermal stability test was carried out to analyze the heatresistant capability of phage $\phi \mathrm{pp} 2$ at $\mathrm{pH} 7.5-8.0$. The phage was incubated at $37,50,61,70$, and $80^{\circ} \mathrm{C}$ for one hour, respectively. As Figure 2 shows, the phage titers at different time intervals demonstrated that phage $\phi p p 2$ stock solution retained almost $100 \%$ infection activity after incubation at temperatures lower than $37^{\circ} \mathrm{C}$ for one hour. When the temperatures rose above $50^{\circ} \mathrm{C}$, viability of phage $\phi p p 2$ declined; about $60 \%$ phages remained alive after being heated for 60 minutes. At temperatures over $60^{\circ} \mathrm{C}$, nearly all phages were inactivated after 15 minutes of incubation.

\section{Genome organization and annotation}

The genome sequence of Vibrio phage фpp2 was determined using the Roche Genome Sequencer system (454
Life Sciences, Branford, CT). A total of 21,452 reads and $7,985,781$ bases, with an average length of 372.3 bases, were obtained. After de novo assembly among at least 40 nucleotide overlap with minimum overlap identity of $90 \%$, the whole genome was aligned to one single contig, with coverage of 32-fold and the $\mathrm{Q}_{40}$ Plus Bases of $98.89 \%$ (where $\mathrm{Q}_{40}$ represents an error rate of 99.99\%). Currently, the draft genome has a total of 246,421 bp, which includes 270 nt of $\mathrm{Q}_{39}$ Minus Bases (0.11\%). The GenBank access number for this new genome is assigned to be JN849462.

The genome size of the Vibrio phage $\phi p p 2$ is $1587 \mathrm{bp}$ larger than 244,834 bp of KVP40 bp and far bigger than the 168,903 bp of $T 4$, while its average $G+C$ content was $42.55 \%$, which is the same as the $42.60 \%$ of KVP40 but not as the $35.3 \%$ of T4. No rRNA genes of the draft assembly were identified using RNAmmer. Sixty tRNA overlap genes that were preliminarily predicted by the RAST were further verified to be 30 using tRNAscan-SE. In annotation for protein coding regions, 30 subsystem features were predicted by the SEED-RAST server, including 15 features which were relevant to phage structure proteins, 2 for phage DNA synthesis, 7 for nucleotide reactions, and one each for fluoroquinolone resistance, protein degradation and RNA metabolism. One possible gene for resistance of beta-lactamase was not included by the auto-annotation.

\section{Large indels (insertion/deletions)}

Overall of Vibrio phage фpp2 was similar to the genome organization of vibriophage KVP40 and Enterobacteria phage T4 (Table 1). In comparison with KVP40, 15 deletions and 19 insertions were found in $\phi p p 2$, of which 25 indels only affected one single ORF. It is noteworthy that a single deletion occurred in the segD-type HE (PEG145 of KVP40), at the junction of KVP40.0145 (at 84923..85078) and KVP40.0146 (complement 85073..85768), implying that \$pp2 had lost this HE. Most of the indel sizes were in the range around 100-400 nt; nevertheless, some large replacements existed, i.e., $621 \mathrm{nt}$ at KVP40.0102 (61372..61992), $702 \mathrm{nt}$ at KVP40.0121 (70639..71307), $687 \mathrm{nt}$ at KVP40.0147 (85926..86240), 664 nt at KVP40.0172 (98546..98713), 672 nt at KVP40.0277 (nrdA, 146553..148778), and $693 \mathrm{nt}$ at KVP40.0315 (178766..178930). Additionally, three KVP40 genes were replaced by giant inserts in фpp2: $1098 \mathrm{nt}$ of фpp2 replaced the gene near KVP40.0363 (gp23, 224506..226050, $1545 \mathrm{nt}), 1143 \mathrm{nt}$ of $\phi \mathrm{pp} 2$ replaced the gene at KVP40.0263 (137878..138114, 237 nt), and 3330 nt of \$pp2 replaced the gene at KVP40.0297 (complement 160413..160988,576 nt). The three giant indels signified that the Vibrio phage $\phi p p 2$ was a new species from KVP40.

\section{Gene functions}

With the extracting plausible protein sequences encoded by the genomic DNAs, 383 PEGs were found in Vibrio 


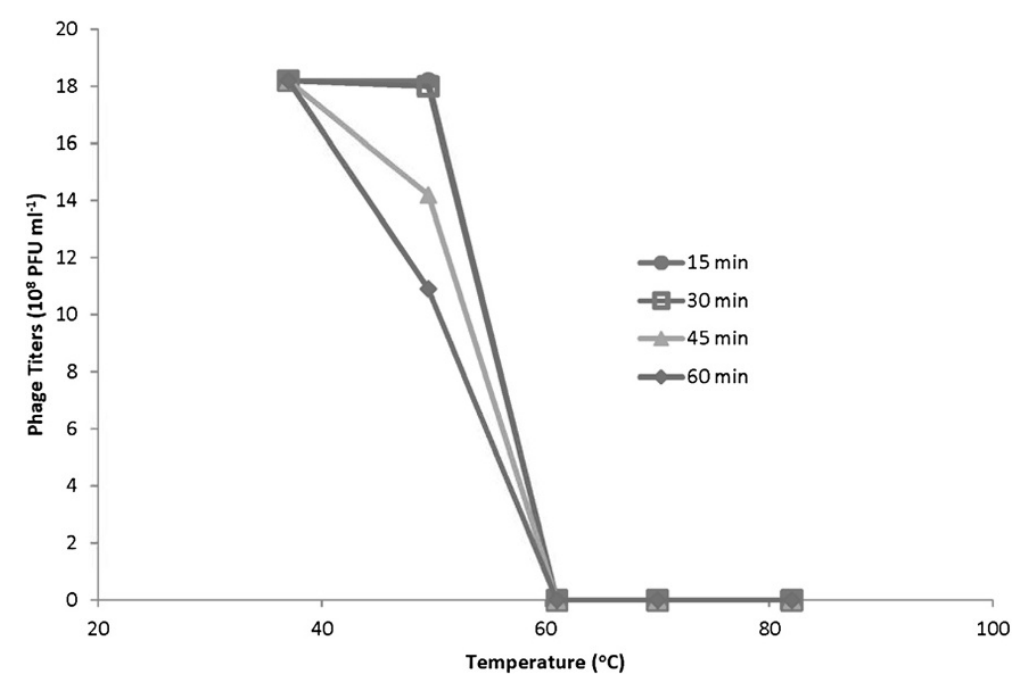

Figure 2 Thermal stability tests of the phage ppp2. Samples were taken at different time intervals to titer the phage particles of infectivity.

phage $\phi p p 2$, in contrast to 381 PEGs for KVP40 found with the same RAST method (Table 1). Functions were identified by sequence similarity (Table 1$) .104(27.2 \%)$ out of 383 PEGs were matched to known functions of T4-like phage genes and assorted bacteria genomes, while functions of 279 (72.8\%) PEGs were still unknown. Among these, as Figure 3 and Table 1 show, 67 PEGs were matched to both T4 and KVP40 (green arrows), 29 PEGs to KVP40 alone (yellow ticks), 7 PEGs to other T4-like (purple and red ticks), and one to assorted bacteria (cyan). Between the genomes of $\phi p p 2$ and KVP40, the similarity of 254 genes was greater than $94 \%$, whereas 17 PEGs of KVP40 and 21 PEGs of $\phi p p 2$ were unmatched to any known, in addition to 15 genes with lower similarity (Additional file 1). At least 29 PEGs (7.6\%) were directly related to phage particle structures, such as head, tail, and baseplate. $\phi p p 2$ uniquely possessed the proteins involved in potassium channel, gp2 (DNA end protector), tRNA nucleotidyltransferase, and mob-type HEs, which were not reported in the case of KVP40. Several genes were split: in $\phi p p 2$, PEG297 shared paralogs with PEG296, as the same pattern for PEG119 sharing with PEG274, while KVP40.0089 (54956..55189) and KVP40.0090 (55200..56117) paralogs were matched to one single $\phi$ pp2 PEG88.

\section{Transfer RNAs}

The RAST predicted 60 pieces of potential tRNAs, spanning in the range of $9175 \mathrm{bp}$ in Vibrio phage $\phi p p 2$, while in KVP40 29 tRNAs were found in the range of $8702 \mathrm{bp}$. Using tRNAscanSE to recalculate the structures with overlapping sequences, 30 tRNAs in the cluster were double verified for Vibrio phage $\phi p p 2$ while 29 tRNAs remained for KVP40; both contained three pseudo-forms of low score for GCA (two) and TGC (one) anticodons. The Vibrio phage $\phi \mathrm{pp} 2$ tRNA cluster encoded for 17 amino acid codons, but there were no anticodons for alanine, glutamine, and tyrosine. The KVP40 tRNA region was 475 bp shorter than the $\phi p p 2$ but shared $97 \%$ similarity over the cluster. A big insert of $465 \mathrm{nt}$ in the middle of the cluster created no putative tRNA structure in the range of insert. In the Vibrio phage $\phi \mathrm{pp} 2$, one extra met-tRNA, which formed from the 28 nt mutation out of $72 \mathrm{nt}$, was created at the upstream of junction that was 6 nt upstream from the 465-nt insert.

\section{Searching mob-like genes and neighbors}

In sequence similarity analysis by PSI-BLAST, three paralog genes of homing endonucleases were found in the Vibrio phage $\phi p p 2:$ PEG79, PEG119, and PEG274, in which the number of amino acid residues was 209, 234, and 224 aa, respectively. The PEG119 and PEG274 were aligned to neighborhood of T4 MobE and close to MobD (Figure 4A). The PEG79 were situated next to the group of MobA (Figure 4A). The PEG119 shared 37\% similarity with PEG274, while PEG79 shared $27 \%$ and $35 \%$ with the other two in pair-wide alignment of amino acid sequences. In Bootstrap analysis with 1000 replicates, the branch percentage showed that the three PEGs in $\phi p p 2$ were all Mob-like homing endonucleases, least likely to be a GIY-YIG type (Figure 4B). Although low overall similarity was found between them, all three PEGs aligned the $\mathrm{H}-\mathrm{N}-\mathrm{H}$ motif very well in their $\mathrm{N}$ termini (Figure 4C). First two His-32 and His-33 in PEG79 were highly conserved within the motif of ExHHILPK for PEG119 and PEG274. The second Asn50 of PEG79 was situated in the motif of SDExNLV, and the third His was paired as HxxxH found in the motif of LTARE $\underline{H}-\underline{H} x L L x K$. 
Table 1 Gene functions of the Vibrio phage $\varphi p p 2$

\begin{tabular}{|c|c|c|c|c|c|c|c|}
\hline Feature ID & Start & Stop & nt (bp) & aa & Function & Match to & Color \\
\hline fig 75320.3.peg.1 & 41 & 973 & 933 & 311 & RNaseH ribonuclease & KVP40 \& T4 & $\mathrm{G}^{*}$ \\
\hline fig 75320.3.peg.3 & 1318 & 1611 & 294 & 98 & late promoter transcription accessory protein & KVP40 & Y \\
\hline fig 75320.3.peg.6 & 3216 & 3761 & 546 & 182 & Frd dihydrofolate reductase & KVP40 \& T4 & G \\
\hline fig 75320.3.peg.7 & 3758 & 4477 & 720 & 240 & ATP-dependent Clp protease proteolytic subunit (EC3.4.21.92) & KVP40 & Y \\
\hline fig 75320.3.peg.8 & 4544 & 5644 & 1101 & 367 & Phage recombination protein & KVP40 \& T4 & G \\
\hline fig 75320.3.peg.10 & 6060 & 7343 & 1284 & 428 & DNA primase-helicase subunit & KVP40 \& T4 & G \\
\hline fig 75320.3.peg.12 & 7583 & 9418 & 1836 & 612 & Ribonucleotide reductase of class III (anaerobic), large subunit (EC 1.17.4.2) & KVP40 \& T4 & G \\
\hline fig 75320.3.peg.15 & 10506 & 10982 & 477 & 159 & Ribonucleotide reductase of class III (anaerobic), activating protein (EC 1.97.1.4) & KVP40 \& T4 & G \\
\hline fig 75320.3.peg.16 & 10982 & 11506 & 525 & 175 & putative serine/threonine protein phosphatase & KVP40 & Y \\
\hline fig 75320.3.peg.18 & 12420 & 13244 & 825 & 275 & 98.1\% KVP40 DNA helicase, phage-associated & KVP40 & Y \\
\hline fig 75320.3.peg.19 & 13244 & 13720 & 477 & 159 & gp61.1 conserved hypothetical & KVP40 \& T4 & G \\
\hline fig 75320.3.peg.20 & 13801 & 14859 & 1059 & 353 & DNA primase (EC 2.7.7.-)/DNA helicase (EC 3.6.1.-). Phage-associated & KVP40 \& T4 & G \\
\hline fig 75320.3.peg.21 & 14859 & 15356 & 498 & 166 & Deoxyuridine 5\&\#39;-triphosphate nucleotidohydrolase (EC 3.6.1.23) & KVP40 & Y \\
\hline fig 75320.3.peg.23 & 15589 & 16281 & 693 & 231 & exonuclease A & KVP40 \& T4 & G \\
\hline fig 75320.3.peg.31 & 18182 & 19081 & 900 & 300 & Thymidylate synthase (EC 2.1.1.45) & KVP40 & G \\
\hline fig 75320.3.peg.42 & 23359 & 24024 & 666 & 222 & NAD-dependent protein deacetylase of SIR2 family & KVP40 & Y \\
\hline fig 75320.3.peg.43 & 24180 & 25973 & 1794 & 598 & DNA gyrase subunit B (EC 5.99.13) & KVP40 \& T4 & G \\
\hline fig 75320.3.peg.49 & 27350 & 28078 & 729 & 243 & Ser/Tar protein phosphatase family protein & KVP40 & Y \\
\hline fig 75320.3.peg.55 & 30282 & 31625 & 1344 & 448 & DNA ligase & KVP40 \& T4 & G \\
\hline fig 75320.3.peg.60 & 36003 & 36242 & 240 & 80 & glutaredoxin & KVP40 & Y \\
\hline fig 75320.3.peg.61 & 36301 & 37197 & 897 & 299 & Phage capsid vertex protein (T4-like gp24) & KVP40 \& T4 & G \\
\hline fig 75320.3.peg.62 & 37206 & 37718 & 513 & 171 & T4-like phage RNA polymerase sigma factor for late transcription \# T4-like phage gp55\#T4GC0140 & KVP40 \& T4 & G \\
\hline fig 75320.3.peg.68 & 41040 & 41504 & 465 & 155 & gp30.3 & KVP40 \& T4 & G \\
\hline fig 75320.3.peg.69 & 41509 & 41994 & 486 & 162 & Putative 5\&\#39;(3\&\#39;)-deoxyribonucleotidase (EC 3.1.3.-) & KVP40 & Y \\
\hline fig 75320.3.peg.70 & 41991 & 43031 & 1041 & 347 & Phage recombination-related endonuclease Gp47 & KVP40 \& T4 & G \\
\hline fig 75320.3.peg.72 & 43248 & 45485 & 2238 & 746 & recombination endonuclease subunit & KVP40 \& T4 & G \\
\hline fig 75320.3.peg.75 & 46247 & 46912 & 666 & 222 & $27.11 \%$ T4 Sliding clamp DNA polymerase accessory protein, phage associated \# Gp45 & T4 & DG \\
\hline fig 75320.3.peg.76 & 46983 & 47939 & 957 & 319 & Replication factor C small subunit/Phage DNA polymerase clamp loader subunit \# T4-like phage gp44 \# T4 GC0157 & KVP40 \& T4 & G \\
\hline fig 75320.3.peg.77 & 47950 & 48438 & 489 & 163 & 31.11\% T4 Phage DNA polymerase clamp loader (fig|10665.1.peg.49) & T4 & DG \\
\hline fig 75320.3.peg.78 & 48473 & 48853 & 381 & 127 & RegA translaticnal repressor of early genes & KVP40 \& T4 & G \\
\hline fig 75320.3.peg.79 & 49482 & 48856 & 627 & 209 & MobE homing endonuclease & $\mathrm{T} 4$ & $\mathbf{R}$ \\
\hline fig 75320.3.peg.81 & 50130 & 52682 & 2553 & 851 & DNA polymerase & KVP40 \& T4 & G \\
\hline
\end{tabular}




\begin{tabular}{|c|c|c|c|c|c|c|c|}
\hline fig 75320.3.peg.83 & 53047 & 54192 & 1146 & 382 & $\mathrm{Rn} 1 \mathrm{~A}$ & KVP40 \& T4 & G \\
\hline fig 75320.3.peg.88 & 55585 & 56742 & 1158 & 386 & 3\&\#39;-phosphatase, 5\&\#39;-polynucleotide kinase, phage-associated \#T4-like phage Pset \#T4 GC1648 & KVP40 \& T4 & G \\
\hline fig 75320.3.peg.116 & 69766 & 70218 & 453 & 151 & CMP/dCMP deaminase, zinc-binding & KVP40 \& T4 & G \\
\hline fig 75320.3.peg.117 & 70273 & 71199 & 927 & 309 & NADPH-dependent 7-cyano-7-deazaguanine reductase (EC 1.7.1.-) & KVP40 & Y \\
\hline fig 75320.3.peg.118 & 71268 & 71942 & 675 & 225 & GTP cyclohydrolase I (EC 3.5.4.16) type 1 & KVP40 & Y \\
\hline fig 75320.3.peg.119 & 72615 & 71914 & 702 & 234 & Phage-associated homing endonuclease & T4 & $\mathbf{R}$ \\
\hline fig 75320.3.peg.121 & 74083 & 74991 & 909 & 303 & NADPH-dependent 7-cyano-7-deazaguanine reductase (EC 1.7.1.-) & KVP40 & Y \\
\hline fig 75320.3.peg.122 & 75047 & 75763 & 717 & 239 & Queuosine Biosynthesis QueC ATPase & KVP40 & Y \\
\hline fig 75320.3.peg.124 & 76380 & 76655 & 276 & 92 & $32.1 \%$ T4 Phage tail fibers & T4 & DG \\
\hline fig 75320.3.peg.127 & 77160 & 77498 & 339 & 113 & Phage capsid and scaffold & KVP40 & Y \\
\hline fig 75320.3.peg.129 & 78455 & 77946 & 510 & 170 & gp49 recombination endonuclease VII & KVP40 \& T4 & G \\
\hline fig 75320.3.peg.131 & 78799 & 79806 & 1008 & 336 & RNA ligase, phage-associated & KVP40 \& T4 & G \\
\hline fig 75320.3.peg.141 & 83194 & 85263 & 2070 & 690 & Phage rllA lysis inhibitor & KVP40 \& T4 & G \\
\hline fig 75320.3.peg.142 & 85256 & 86293 & 1038 & 346 & rllB protector from prophage-induced early lysis & KVP40 \& T4 & G \\
\hline fig 75320.3.peg.148 & 89179 & 90216 & 1041 & 347 & NrdC 11 conserved hypothetical protein. & KVP40 \& T4 & G \\
\hline fig 75320.3.peg.151 & 90946 & 92211 & 1266 & 422 & Dda DNA helicase & KVP40 \& T4 & G \\
\hline fig 75320.3.peg.157 & 94752 & 95777 & 1026 & 342 & Nicotinamide-nucleotide adenylyltransferase, NadM family (EC 2.7.7.1)/ADP-ribose pyrophosphatase & KVP40 & Y \\
\hline fig 75320.3.peg.188 & 105782 & 106366 & 585 & 195 & Thymidine kinase (EC 2.7.1.21) & KVP40 \& T4 & G \\
\hline fig 75320.3.peg.201 & 111295 & 111711 & 417 & 139 & endonuclease & KVP40 \& T4 & G \\
\hline fig 75320.3.peg.208 & 113557 & 114537 & 981 & 327 & Nicotinamide-nucleotide adenyly|transferase, NadR family (EC 2.7.7.1)/Ribosyln:cotinamide kinase (EC 2.7.1.22) & KVP40 & Y \\
\hline fig 75320.3.peg.212 & 115492 & 116157 & 666 & 222 & Ribosyl nicotinamide transporter, PnuC-like & KVP40 & Y \\
\hline fig 75320.3.peg.249 & 130608 & 131138 & 531 & 177 & Cell wall mannoprotein with similarity to Tir1p, Tir2p, Tir3p, and Tir4p; expressed under anaerobic conditions, comp & KVP40 & Y \\
\hline fig 75320.3.peg.255 & 134377 & 135366 & 990 & 330 & moa A/nifB/pqqE family protein & KVP40 & Y \\
\hline fig 75320.3.peg.256 & 136483 & 135359 & 1125 & 375 & moa A/nifB/pqqE family protein & KVP40 & Y \\
\hline fig 75320.3.peg.260 & 138690 & 140183 & 1494 & 498 & Nicotinamide phosphoritosyltransferase (EC 2.4.2.12) & KVP40 & Y \\
\hline fig 75320.3.peg.268 & 144971 & 143919 & 1053 & 351 & moaA/nifB/pqqE family protein & KVP40 & Y \\
\hline fig 75320.3.peg.273 & 147028 & 149253 & 2226 & 742 & Ribonucleotide reductase of class la (aerobic), alpha subunit (EC 1.17.4.1) & KVP40 \& T4 & G \\
\hline fig 75320.3.peg.274 & 149293 & 149964 & 672 & 224 & Phage-associated homing endonuclease & T4 & $R$ \\
\hline fig 75320.3.peg.275 & 149957 & 151081 & 1125 & 375 & Ribonucleotide reductase of class la (aerobic), beta subunit (EC 1.17.4.1) & KVP40 \& T4 & G \\
\hline fig 75320.3.peg.276 & 151083 & 151382 & 300 & 100 & NrdC thioredoxin & KVP40 \& T4 & G \\
\hline fig 75320.3.peg.279 & 152313 & 153317 & 1005 & 335 & Thioredoxin, phage-associated & KVP40 \& T4 & G \\
\hline fig 75320.3.peg.280 & 153363 & 154649 & 1287 & 429 & gp52 topoisomerase II medium subunit & KVP40 \& T4 & G \\
\hline fig 75320.3.peg.282 & 154900 & 155781 & 882 & 294 & Queuosine Biosynthesis QueE Radical SAM & KVP40 & Y \\
\hline
\end{tabular}


Table 1 Gene functions of the Vibrio phage ppp2 (Continued)

\begin{tabular}{|c|c|c|c|c|c|c|c|}
\hline fig 75320.3.peg.293 & 160936 & 161235 & 300 & 100 & anti-sigma70 protein & KVP40 & Y \\
\hline fig 75320.3.peg.296 & 165535 & 162206 & 3330 & 1110 & Phage tail fibers (Match to KVP40 peg.297) & KVP40 & Y \\
\hline fig 75320.3.peg.297 & 168882 & 165607 & 3276 & 1092 & tail fiber fragment & KVP40 & Y \\
\hline fig 75320.3.peg.322 & 184667 & 184212 & 456 & 152 & gp57B conserved hypothetical protein & KVP40 \& T4 & G \\
\hline fig 75320.3.peg.324 & 185581 & 184943 & 639 & 213 & dNMP kinase & KVP40 \& T4 & G \\
\hline fig 75320.3.peg.325 & 186347 & 185814 & 534 & 178 & gp3 tail completion and health stabilizer protein & KVP40 \& T4 & G \\
\hline fig 75320.3.peg.328 & 189332 & 188484 & 849 & 283 & Phage baseplate hub & KVP40 \& T4 & G \\
\hline fig 75320.3.peg.329 & 190090 & 189344 & 747 & 249 & Phage baseplate-tail tube initiator & KVP40 & Y \\
\hline fig 75320.3.peg.330 & 190507 & 190094 & 414 & 138 & 55.97\% T4 Phage DNA end protector during packaging & T4 & DG \\
\hline fig 75320.3.peg.331 & 191144 & 190689 & 456 & 152 & Phage head completion protein & KVP40 \& T4 & G \\
\hline fig 75320.3.peg.332 & 191211 & 192350 & 1140 & 380 & Phage baseplate tail tube cap & KVP40 \& T4 & G \\
\hline fig 75320.3.peg.333 & 192350 & 192928 & 579 & 193 & Phage baseplate wedge & KVP40 \& T4 & G \\
\hline fig 75320.3.peg.335 & 194207 & 195466 & 1260 & 420 & Phage baseplate hub & KVP40 \& T4 & G \\
\hline fig 75320.3.peg.337 & 195956 & 196252 & 297 & 99 & PAAR & KVP40 \& T4 & G \\
\hline fig 75320.3.peg.340 & 197717 & 198136 & 420 & 140 & Phage baseplate wedge & KVP40 \& T4 & G \\
\hline fig 75320.3.peg.341 & 198223 & 200181 & 1959 & 653 & Phage baseplate wedge & KVP40 \& T4 & G \\
\hline fig 75320.3.peg.342 & 200181 & 203678 & 3498 & 1166 & Phage baseplate wedge initiator & KVP40 \& T4 & G \\
\hline fig 75320.3.peg.343 & 203680 & 204702 & 1023 & 341 & Phage baseplate wedge & KVP40 \& T4 & G \\
\hline fig 75320.3.peg.344 & 204758 & 205714 & 957 & 319 & gp9 & KVP40 \& T4 & G \\
\hline fig 75320.3.peg.345 & 205724 & 207970 & 2247 & 749 & Phage baseplate wedge & KVP40 \& T4 & G \\
\hline fig 75320.3.peg.348 & 210191 & 211612 & 1422 & 474 & prophage LambdaSa04, minor structural protein, putative & KVP40 \& T4 & G \\
\hline fig 75320.3.peg.349 & 211911 & 213590 & 1680 & 560 & Phage neck whiskers & KVP40 \& T4 & G \\
\hline fig 75320.3.peg.350 & 213601 & 214524 & 924 & 308 & Phage neck protein & KVP40 \& T4 & G \\
\hline fig 75320.3.peg.351 & 214528 & 215364 & 837 & 279 & Phage neck protein & KVP40 \& T4 & G \\
\hline fig 75320.3.peg.352 & 215593 & 216726 & 1134 & 378 & tail health stabilizer and completion protein & KVP40 \& T4 & G \\
\hline fig 75320.3.peg.354 & 217441 & 217989 & 549 & 183 & Phage terminase, small subunit & KVP40 \& T4 & G \\
\hline fig 75320.3.peg.355 & 217949 & 219751 & 1803 & 601 & Phage terminase, large subunit & KVP40 \& T4 & G \\
\hline fig 75320.3.peg.356 & 219798 & 221813 & 2016 & 672 & Phage tail health monomer & KVP40 \& T4 & G \\
\hline fig 75320.3.peg.357 & 221864 & 222364 & 501 & 167 & Phage tail fibers & KVP40 \& T4 & G \\
\hline fig 75320.3.peg.358 & 222404 & 223951 & 1548 & 516 & portal vertex protein of head & KVP40 \& T4 & G \\
\hline fig 75320.3.peg.360 & 224131 & 224622 & 492 & 164 & Phage capsid and scaffold & KVP40 \& T4 & G \\
\hline fig 75320.3.peg.361 & 224625 & 225266 & 642 & 214 & Phage prohead core scaffold protein and protease & KVP40 \& T4 & G \\
\hline fig 75320.3.peg.362 & 225299 & 226141 & 843 & 281 & Phage scaffold prohead core protein & KVP40 \& T4 & G \\
\hline
\end{tabular}


Table 1 Gene functions of the Vibrio phage ppp2 (Continued)

\begin{tabular}{|c|c|c|c|c|c|c|c|}
\hline fig 75320.3.peg.363 & 226212 & 227756 & 1545 & 515 & Phage major capsid protein & KVP40 \& T4 & G \\
\hline fig 75320.3.peg.364 & $\underline{228910}$ & $\underline{227813}$ & $\underline{1098}$ & $\underline{366}$ & $\underline{\text { PSI-BLAST tRNA nucleotidyltransferase (Acinetobacter baumannii \& Pseudomonas fluorescens) }}$ & $\underline{\text { KVP40 \& T4 }}$ & $\underline{G}$ \\
\hline fig 75320.3.peg.367 & 230824 & 231315 & 492 & 164 & Inh & KVP40 \& T4 & G \\
\hline fig 75320.3.peg.377 & 236908 & 238431 & 1524 & 508 & DNA helicase, phage-associated & KVP40 \& T4 & G \\
\hline fig 75320.3.peg.381 & 239752 & 239339 & 414 & 138 & UvsY recombination, repair and ssDNA binding protein & KVP40 & Y \\
\hline fig 75320.3.peg.383 & 246382 & 242612 & 3771 & 1257 & gp34 long tail fiber, proximal subunit & KVP40 & Y \\
\hline
\end{tabular}

The legends in the last column represented by G, Y, P and R correspond to the real color shown in Genome map of the Vibrio phage $\varphi p p 2$ (Figure 3). G (green arrows in Figure 3) indicates the gene functions were matched to both Enterobacteria phage T4 and Vibrio phage KVP40. Shallow lines with Y (yellow and ticks on the circle in Figure 3) indicate the gene functions fitted to KVP40 only. Bold $\mathbf{P}$ and italic bold $\boldsymbol{R}$ (purple and red bars in Figure 3) represent the genes which only aligned well with T4; additionally, the italic bold $R$ (red bars with the number in Figure 3 ) indicates the PEG numbers of potential HE. The underlining (cyan in Figure 3) demonstrates one gene that matched to assorted bacteria. 


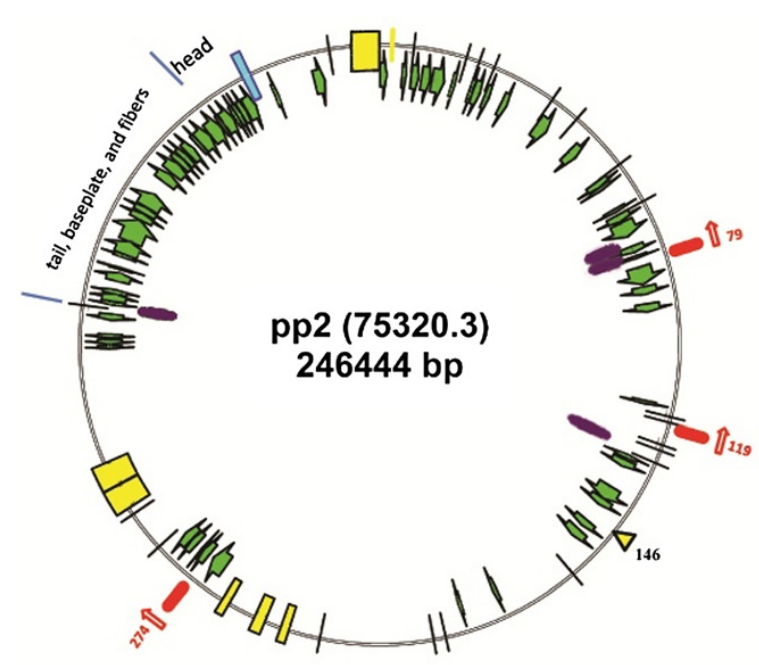

Figure 3 Genome map of the Vibrio phage $\varphi p p 2$. Green arrows indicate the genes matched to both Enterobacteria phage T4 and Vibrio phage KVP40. Yellow ticks on the circle indicate that the genes fitted to KVP40 only while the yellow triangle indicates the absent site for KVP40.0146 HE gene. Purple represents that the genes only aligned well with T4. The cyan is for one gene matched to GTP cyclohydrolase I from Bdellovibrio bacteriovorus HD100, Vibrio angustum S14, and Cytophaga hutchinsonii ATCC 33406. Red bars with the number indicate the PEG numbers of potential HE.

We identified the Mob types of $\phi p p 2$ around the genome according to the orientation similarity to the neighbor ORFs of 15 homing endonucleases in Enterobacteria phage T4: gt (glucosyl transferase) and nrd (ribonucleotide reductase) orthologs. The details of the search methods are described in Additional file 2. No match to T4 $\alpha$.gt or $\beta$.gt was found in entire genomes of $\phi p p 2$ and KVP40 (NC_005083); therefore, the mobB-like gene could not exist in $\phi p p 2$. Four $n r d$-like genes were found in фpp2: one was found explicitly by the RAST and three others were implicit but manually confirmed with PSIBLAST searches. The PEG12 protein was similar to the large subunit of anaerobic ribonucleotide reductase of class III (EC 1.17.4.2), with $52.05 \%$ similarity to T4 nrdG, while PEG15 was assumed to be the activating protein (EC 1.97.1.4) for the ribonucleotide reductase with $52.74 \%$ similarity to T4 $n r d D$. PEG132, which was matched to T4p232 in the boundary of MobE and downstream close-by $\operatorname{seg} \mathrm{D}$, was denoted as $n r d \mathrm{~B} .1$. The fourth $n r d$-like gene, 1041 nt of PEG148 (347 aa, 89176..90216) in $\phi p p 2$, was mapped to T4 nrdC.11.

Using the neighbor-indirect method (details in Additional file 2), the neighbors of T4 mob genes were mapped back to $\phi p p 2$ genome. The neighbor gene T4p074 (nrdG) of mobC (T4p075) was back-projected to фpp2 PEG15 with a similarity of 52.05\%; another neighbor gene T4p076 ( $n r d \mathrm{D})$ was matched to $\phi p p 2$ PEG12 with a similarity of $52.74 \%$. The distance of the PEG12/ 15 pair was at least 37874 nt apart from the PEG79 - it was even farther to PEG 119 and PEG274. Similarly, the PEG132 and PEG148 were still too far to be adjunction neighbors for all three potential $\phi p p 2 m o b$ genes, i.e., PEG132 was 7895 nt apart from PEG119.

Alternatively, using the so-called neighbor-direct method (Additional file 2), the mob-neighbor genes of фpp2 PEG79, PEG119, and PEG274 were manually de novo searched with PSI-BLAST. Neither neighbors of PEG79 (upstream PEGs $70 \sim 78$ and downstream PEGs $80 \sim 90$ ) nor of PEG119 (upstream PEGs $110 \sim 117$ and downstream PEGs $120 \sim 125)$ were in any way close to $n r d$-like genes (Figure 5A and B).

\section{Vibrio phage $\varphi$ pp2 PEG274 with mobE-type neighbors}

In de novo identification of a mob-type for $\phi \mathrm{pp} 2$ PEG274 (672 nt) using the neighbor-direct method, фpp2 PEG273 (2226 nt) of the upstream neighbor gene was blasted to NrdA of Aeromonas phages (PX29 and phiAS5), Enterobacteria phages (JSE, RB49, phi1, and T4), and Shigella phage SP18 (Figure 5C). The downstream neighbor PEG275 (1125 nt) was blasted to NrdB of Aeromonas phages phiAS5 and Aeh1, Klebsiella phage $\mathrm{KP} 15$, and Enterobacteria phage RB16. Another neighbor, PEG276 (300 nt), was also blasted to the NrdC thioredoxin; it aligned well as $86 \%$ homologous to $\mathrm{NrdC}$ thioredoxin in Aeromonas phages phiAS5, Aeh1, and 65, as well as to Klebsiella phage KP15, Shigella phage SP18, and Enterobacteria phages RB16, RB43, and ime09. With the matches of upstream and downstream of $n r d$-like genes which complemented the full organization of MobE neighbors, the фpp2 PEG274 can be annotated as a MobE-type $\mathrm{HE}$, without the existence of I-TevIII intron.

\section{Expression of $\varphi$ pp2 PEG 274 gene}

All homing endonucleases of $\phi p p 2$ and KVP40 started with an AUG initiation codon. For фpp2 PEG 274, AGGA as a ribosome binding site (RBS) was optimally situated 6 nt upstream of the PEG start codon while translation initiation regions are not positioned at the optimal distance of 6-9 nucleotides from the AUG codon for PEG79 and PEG 119. The AAGAGAG for PEG79 was not a good match to antisense of small rRNA while the predicted PEG119 RBS AGGA is immediately adjunct to the AUG codon, which is rarely considered to be a good initiation site for translation.

As shown in Figure 3, the direction of transcription for PEG79 and PEG119 was counter-clockwise, whereas the PEG274 promoter was clockwise, which was the same as most T4 homolog genes. The NNPP predicted several promoters of high scores $(>0.95)$ in the upstream of these three genes. It is worth noting that three 


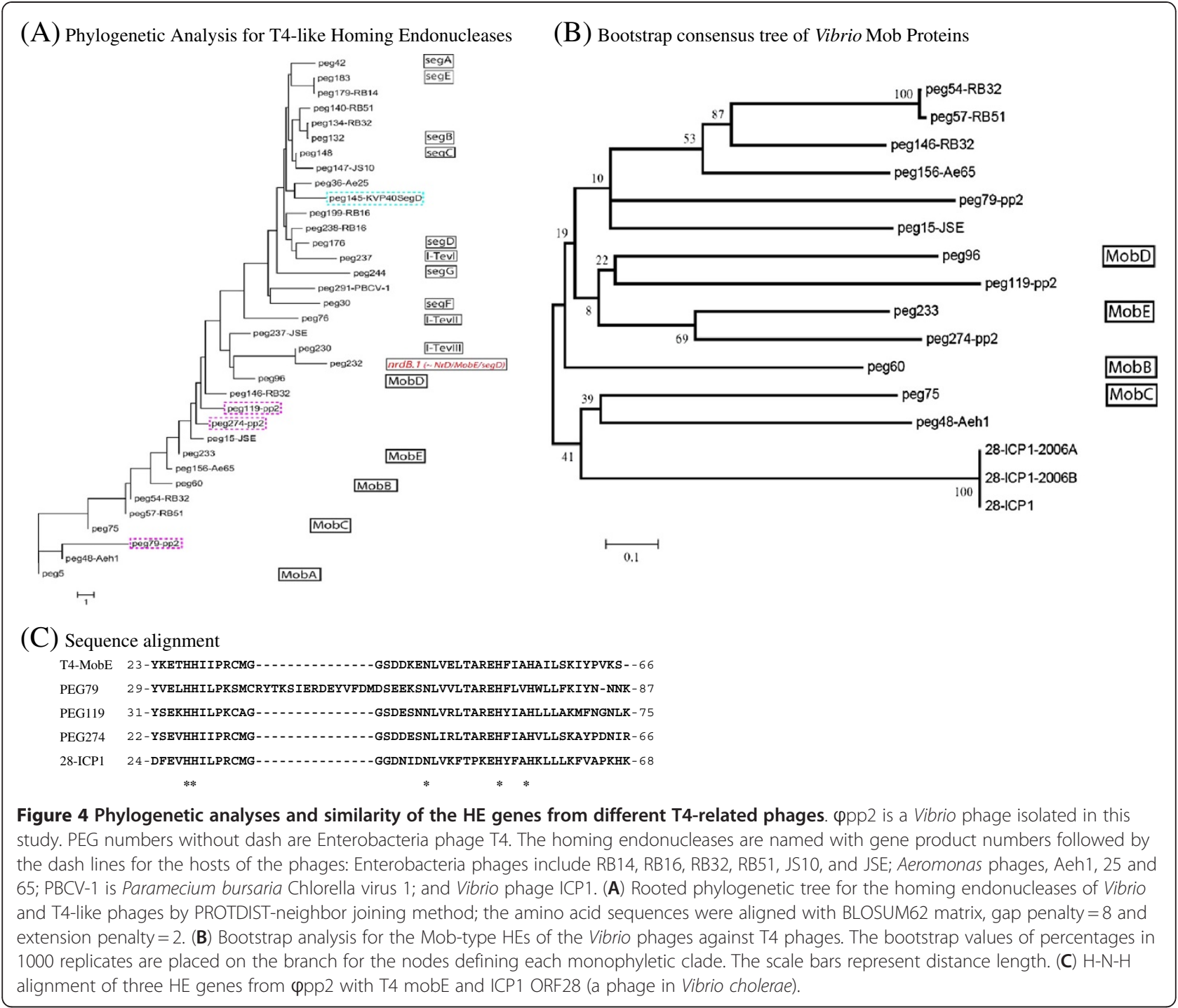

promoters were identified around PEG274, the aforementioned MobE-type homing endonuclease. In contrast to the translational initiation AUG position of PEG274 at 149293-149964 in the genome of $\phi p p 2$, the nearby promoters were also positioned at 148783 (510 nt upstream; pR148783), 149272 (21 nt immediately upstream; pR149272), and 149974 (10 nt downstream; pR149974). pR149272 was the best fit to the promoter consensus, which consisted of TTGTGA for -35 box and ATGTAAAAT for -10 box. Accompanying this promoter, some weak binding sites for transcription factors were also observed: TGTAAAAT for rpoD17 at position 149258, ATATAAAT for argR2at 149264, and GTTCATAT for torR at 149273.

\section{Discussion}

Electron microscopy revealed that the phage $\phi p p 2$ particles were morphologically similar to $\mathrm{T} 4$ phage and vibriophage KVP40, which is a long head ( 140 nm long and $\sim 70 \mathrm{~nm}$ wide) with a prolate icosahedral capsid and a contractile tail with associated baseplate and extended tail fibers. $\phi \mathrm{pp} 2$ is most likely type A phage in Bradley's classification of Myoviridae [38], based on the morphological characteristics (Figure 1). The protein profiles in фpp2 contain a heavy band of $\sim 50 \mathrm{kD}$, which is similar to known T4 structure proteins of major capsid protein (data not shown). With hourly heat-tolerance at $50^{\circ} \mathrm{C}$ (Figure 2), this phage could infect aquaculture pathogens, $V$. parahaemolyticus, $V$. damsela and $V$. alginolyticus. The complete genome of the new Vibrio phage фpp2 was sequenced (GenBank access_no JN849462), which was a sibling phage of KVP40 but with different HE genes (Figure 3).

In the phylogenetic tree (Figure 4A), the PEG79 was distantly situated next to the group of MobA. Although their overall similarity was low, the $\mathrm{N}$-termini of all 


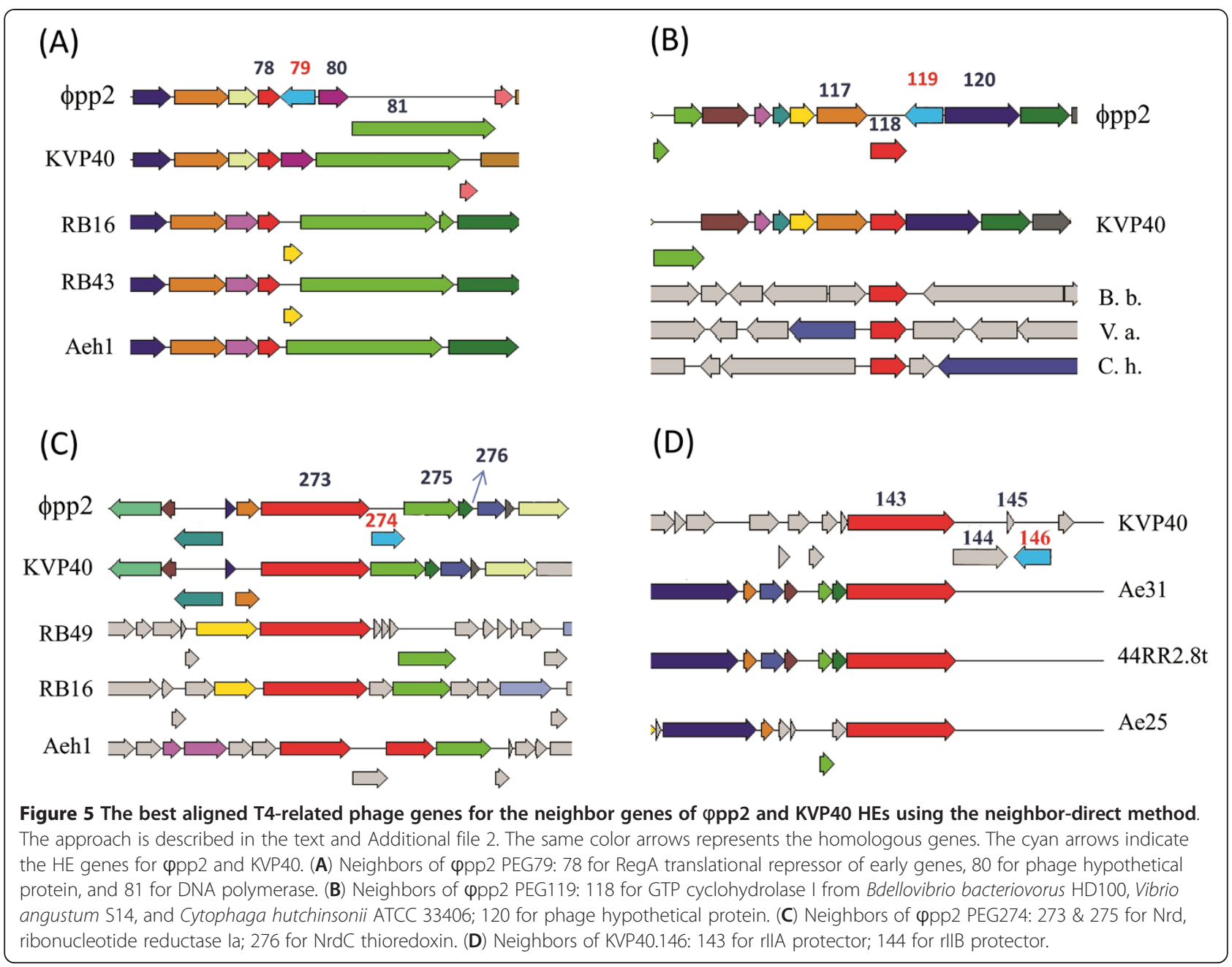

three PEGs aligned well with the $\mathrm{H}-\mathrm{N}-\mathrm{H}$ motif (Figure 4C). The first His-32/33 in PEG79 was highly conserved within the motif of ExHHILPK for PEG119 and PEG274. The second Asn was situated in the motif of SDExNLV and the third His-pair was in the paired form of $\mathrm{HxxxH}$ found in the motif of LTAREHHxLLxK. This reveals that the $\phi p p 2$ HE genes belong to Mob-type because the $\mathrm{H}-\mathrm{N}-\mathrm{H}$ is the critical motif for the enzyme activity [10]. The vibriophage KVP40 carries segD/C (KVP40.0146) [8]. V. cholerae ORF80 in ICP1 belongs to segG (data not shown) while another ICP1ORF28 is closely related to MobC (Figure 5B and C) [9].

By PSI-BLAST directly from the neighbor genes of фpp2 PEG274 (the neighbor-direct method), PEG273, PEG275, and PEG276 were highly homologous to NrdA, $\mathrm{NrdB}$ and NrdC thioredoxin in Aeromonas phages, Enterobacteria phages, Klebsiella phage KP15 and Shigella phage SP18, respectively. With match of both upand downstream, together with the conserved motif of $\mathrm{HE}$ in Figure 4C, the PEG274 can be annotated as MobE-type HE. For PEG274 protein expression, we found a good promoter (pR149272) immediately upstream of the PEG274 gene; thus, the promoter was considered as endonuclease-specific. The transcript of PEG274 mRNA was also equipped with a good consensus of ribosome binding site AGGA at 6 nt upstream of the start codon AUG.

Sequence of ppp2 PEG79 was comparatively similar to MobA gene, but PEG79 was flanked by DNApol and regA (phage endoribonuclease translational repressor of early genes; Figure 5A), where they do not neighbor any mob genes in T4. The PEG119 and PEG79 genes were similar to T4p232 and T4p233 (mobE), respectively. The landmark of T4p131 (e.8, complement 70360..70623) is also very similar to PEG275. In other words, three $\phi p p 2$ mob-like genes (PEG79, PEG119, and PEG274) would be mapped onto the cluster of I-TevIII-nrdB1-mobE located at T4p130 to T4p133 in T4 genome [16, a review]. This implies the characteristics of HE mobility.

KVP40.0146 (696 nt) encoding 231 aa was PSI-BLAST to GIY-YIG endonuclease genes, including Aeromonas phages (phage 25 and phiAS5), Acinetobacter phages 
(Acj61 and Ac42), Chlorella virus FR483, Enterobacteria phages (RB51, RB16, T4), Klebsiella phage KP15, and Staphylococcus phage PH15. As shown in Figure 4A, the phylogenetic analysis plotted KVP40.0146 to be a segC/ $\mathrm{D}$ type. Using the neighbor-direct method (Additional file 2), KVP40.0144 and KVP40.0145 could not be matched to any protein of known function (Figure 5D) while KVP40.142 and KVP40.143 could be similar to rIIA/B lysis protectors. Both were too distant to bracket the KVP40.0146 of GIY-YIG endonuclease gene for mimicking the T4 segD neighbor. In T4 HEs, types of $m o b \mathrm{C}, m o b \mathrm{D}$, and $m o b \mathrm{E}$ can be classified by neighbor elements as well as different arrangements of their promoters: nrdD-mobC-nrdG, mobD-nrdC.11, and nrdA(I-TevIII)-mobE-nrdB, respectively [16]. In KVP40, there are seven $n r d$-like genes that have been identified: $\operatorname{nrdA}, \mathrm{B}, \mathrm{C}, \mathrm{C} .11, \mathrm{D}, \mathrm{G}$, and $\mathrm{H}$. The closest one for KVP40.0146 HE was nrdC.11 (KVP40.0153; 88930..89970), but it was still too distant to be a neighbor of KVP40.0146 to form a good setting as the T4 $m o b C / D / E$. Similarly, four $n r d$ genes were found in Vibrio cholerae ICP1 but without any HE insertion. Therefore, KVP40 and ICP1 did not have the same organization of T4 HEs.

KVP40, sharing the same host as $\phi p p 2$, has only one putative segC/D-type KVP40.0146 (complement 85073..85768), which was also similar in part to T4 $s e g \mathrm{~B} / \mathrm{E}$ and $I-T e v I I I$, even $n r d \mathrm{~B} .1$ [9]. Therefore, the two giant Vibrio phages could partially cross the boundary line at $n r d B .1$ (Figure 4A), in the same host of $V$. parahaemolyticus, to catch the genes and evolve for the future form as the Enterobacteria phage T4 did. The mechanism for the gene exchange and/or evolution may also be similar to the PEG79, PEG119, and PEG 274 in the $\phi p p 2$ as mentioned above.

\section{Conclusions}

In summary, the phage $\phi \mathrm{pp} 2$ was characterized by the morphology, growth, and genomics. In the complete genome sequence analysis in this study, three giant indels and the mobE-type HE signified the Vibrio phage фрp2 to be a new species of T4-related phages, different from KVP40. Our analysis suggested that фpp2 PEG274 was an active mobE gene with transcriptional and translational elements. In the same host, Vibrio parahaemolyticus, the new phage $\phi \mathrm{pp} 2$ can complement its mob-type HE functions with KVP40 that only carries a seg-type HE gene. This spectrum of genome datasets of T4-related Vibrio phages that can co-infect the same host will be useful to investigate the hypothesis that a lateral transfer of freestanding HEs with self-mobility may result in genomic mosaicism by recombining a variety of genetic sequences in phage genomes [18].

\section{Additional files}

Additional file 1: Table S1. The list of genes with low similarity between phages KVP40 and $\varphi$ pp2.

Additional file 2: Text S2. Mob-like gene searches in detail.

\section{Competing interests}

The authors declare that they have no competing interests.

\section{Authors' contributions}

CSL conceived and designed the study. YRL and CSL did the experiments, analyzed the sequence and wrote the manuscript. All authors read and approved the final manuscript.

\section{Acknowledgements}

This research fund is partially supported by the grants from the National Science Council, Taiwan (NSC96-2313-B-110-002-MY3 and NSC99-2313-B-110002-MY3), and the Ministry of Education, Taiwan (NSYSU95 99C031701; the second term of Top University Program: NSYSU 00C030205 and NCHU 100S05-09) under the ATU plan. We thank Professor Long-Huw Lee (National Chung-Hsing University) as the grant organizer of intercampus ATU plan, as well as Chi-Wen Chiu and Feng-Yi Chang in helping initial phage screening, Yu-Tin Liu in helping gel preparations, Professor Y. W. Chiang and Mr. S-C Lin for EM operation, and Kenneth B. Lin and Dr. Simon White for comments and editing.

\section{Received: 10 October 2011 Accepted: 7 May 2012}

Published: 7 June 2012

\section{References}

1. Daniels NA, Mackinnon L, Bishop R, Altekruse S, Ray B, Hammond RM, Thompson S, Wilson S, Bean NH, Griffin PM, Slutsker L: Vibrio parahaemolyticus infection in the United States, 1973-1998. J Infect Dis 2000, 181:1661-1666.

2. Fujino T, Okuno Y, Nakada D, Aoyama A, Fukai K, Mukai T, Ueho T: On the bacteriological examination of Shirasu-food poisoning. Med J Osaka Univ 1953, 4:299-304

3. Levine WC, Griffin PM: Vibrio infections on the Gulf Coast: results of first year of regional surveillance. J Infect Dis 1993, 167:479-483.

4. Morris JG, Black RE: Cholera and other vibrioses in the United States. N Engl J Med 1985, 312:343-350.

5. Johnson DE, Weinberg L, Ciarkowski J, West P, Colwell RR: Wound infection caused by Kanagawa-negative Vibrio parahaemolyticus. J Clin Microbiol 1984 October, 20(4):811-812.

6. Alam M, Chowdhury WB, Bhuiyan NA, Islam A, Hasan NA, Nair GB, Watanabe H, Siddique AK, Huq A, Sack RB, Akhter MZ, Grim CJ, Kam K-M, Luey CKY, Endtz HP, Cravioto A, Colwell RR: Serogroup, virulence, and genetic traits of Vibrio parahaemolyticus in the estuarine ecosystem of Bangladesh. Appl Environ Microbiol 2009, 75:6268-6274.

7. Marano NN, Daniels NA, Easton AN, McShan A, Ray B, Wells JG, Griffin PM, Angulo FJ: A survey of stool culturing practices for Vibrio species at clinical laboratories in Gulf Coast states. J Clin Microbio/ 2000, 38: 2267-2270.

8. Miller ES, Heidelberg JF, Eisen JA, Nelson WC, Durkin AS, Ciecko A Feldblyum TV, White O, Paulsen IT, Nierman WC, Lee J, Szczypinski B, Fraser $\mathrm{CM}$ : Complete genome sequence of the broad-host-range vibriophage KVP40: comparative genomics of a T4-related bacteriophage. J Bacteriol 2003, 185(17):5220-5233.

9. Seed KD, Bodi KL, Kropinski AM, Ackermann H-W, Calderwood SB, Qadri F, Camilli A: Evidence of a dominant lineage of Vibrio cholerae-specific lytic bacteriophages shed by cholera patients over a 10-year period in Dhaka. Bangladesh. mBio 2011, 2(1):e00334-10.

10. Corina LE, Qiu W, Desai A, Herrin DL: Biochemical and mutagenic analysis of I-Crell reveals distinct but important roles for both the $\mathrm{H}-\mathrm{N}-\mathrm{H}$ and GIY-YIG motifs. Nucleic Acids Res 2009, 37(17):5810-5821.

11. Wilson GW, Edgell DR: Phage T4 mobE promotes trans homing of the defunct homing endonuclease I-Tevlll. Nucleic Acids Res 2009, 37(21) :7110-7123.

12. Mueser TC, Hinerman JM, Devos JM, Boyer RA, Williams KJ: Structural analysis of bacteriophage T4 DNA replication: a review in the Virology 
Journal series on bacteriophage T4 and its relatives. Virol J 2010, 7: 359-375.

13. Landthaler M, Shub DA: The nicking homing endonuclease I-Basl is encoded by a group I intron in the DNA polymerase gene of the Bacillus thuringiensis phage Bastille. Nucleic Acids Res 2003, 31:3071-3077.

14. Sharma M, Hinton DM: Purification and characterization of the SegA protein of bacteriophage $\mathrm{T} 4$, an endonuclease related to proteins encoded by group I introns. J Bacteriol 1994, 176:6439-6448.

15. Tseng MJ, He P, Hilfinger JM, Greenberg GR: Bacteriophage T4 nrdA and $n r d B$ genes, encoding ribonucleotide reductase, are expressed both separately and coordinately: characterization of the nrdB promoter. J Bacteriol 1990, 172:6323-6332.

16. Edgell DR, Gibb EA, Belfort M: Mobile DNA elements in T4 and related phages. Virol J 2010, 7:290-304.

17. Eddy SR, Gold L: The phage T4 nrdB intron: a deletion mutant of a version found in the wild. Genes Dev 1991, 5(6):1032-1041.

18. Petrov VM, Ratnayaka S, Nolan JM, Miller ES, Karam JD: Genomes of the T4related bacteriophages as windows on microbial genome evolution. Virol J 2010, 7:292-320.

19. Cabrera-García ME, Vázquez-Salinas C, Quiñones-Ramírez El: Serologic and molecular characterization of Vibrio parahaemolyticus strains isolated from seawater and fish products of the Gulf of Mexico. Appl Environ Microbiol 2004, 70(11):6401-6406.

20. Lin Y-R, Chiu C-W, Chang F-Y, Lin C-S: Characterization of a new phage, termed $\varphi$ A318, which is specific for Vibrio alginolyticus. Arch Viol 2012, xx (in press; DOI 10.1007/s00705-012-1244-8, Feb 11, 2012).

21. Lu M-W, Liu W, Lin C-S: Infection competition against grouper nervous necrosis virus by virus-like particles produced in Escherichia coli. J Gen Virol 2003, 84:1577-1582

22. Sambrook J, Russell DW: Molecular cloning: a laboratory manual. 3rd edition. Cold Spring Harbor, NY: Cold Spring Harbor Laboratory Press; 2001.

23. Shafia F, Thompson TL: Calcium ion requirement for proliferation of bacteriophage Phi Mu-4. J Bacteriol 1964, 88:293-296.

24. Adams MH: Bacteriophages. New York: Interscience; 1959

25. Abedon ST, Herschler TD, Stopar D: Bacteriophage latent-period evolution as a response to resource availability. Appl Env Microbiol 2001, 67: 4233-4241.

26. Mitra S, Basu S: Some biophysical properties of a vibriophage and its DNA. Biochim Biophys Acta 1968, 155(1):143-149.

27. Lind PA, Andersson DI: Whole-genome mutational biases in bacteria. Proc. Natl. Acad. Sci. USA 2008, 105(46):7878-17883.

28. Aziz RK, Bartels D, Best AA, DeJongh M, Disz T, Edwards RA, Formsma K, Gerdes S, Glass EM, Kubal M, Meyer F, Olsen GJ, Olson R, Osterman AL, Overbeek RA, McNeil LK, Paarmann D, Paczian T, Parrello B, Pusch GD, Reich C, Stevens R, Vassieva O, Vonstein V, Wilke A, Zagnitko O: The RAST server: rapid annotations using subsystems technology. BMC Genomics 2008, 9:75-89.

29. Overbeek R, Begley T, Butler RM, Choudhuri JV, Chuang HY, Cohoon M, de Crécy-Lagard V, Diaz N, Disz T, Edwards R, Fonstein M, Frank ED, Gerdes S, Glass EM, Goesmann A, Hanson A, Iwata-Reuyl D, Jensen R, Jamshidi N, Krause L, Kubal M, Larsen N, Linke B, McHardy AC, Meyer F, Neuweger H, Olsen G, Olson R, Osterman A, Portnoy V, Pusch GD, Rodionov DA, Rückert C, Steiner J, Stevens R, Thiele I, Vassieva O, Ye Y, Zagnitko O, Vonstein V: The subsystems approach to genome annotation and its use in the project to annotate 1000 genomes. Nucleic Acids Res 2005, 33(17):5691-702.

30. Delcher AL, Bratke KA, Powers EC, Salzberg SL: Identifying bacterial genes and endosymbiont DNA with glimmer. Bioinformatics 2007, 23 (6):673-679.

31. Lagesen K, Hallin PF, Roland EA, Stafeldt HH, Rognes T, Ussery DW: RNammer: consistent annotation of rRNA genes in genomic sequences. Nucleic Acids Res 2007, 35(9):3100-3108.

32. Lowe TM, Eddy SR: tRNAscan-SE: a program for improved detection of transfer RNA genes in genomic sequence. Nucleic Acids Res 1997, 25: 955-964.

33. Reese MG: Computational prediction of gene structure and regulation in the genome of Drosophila melanogaster, PhD Thesis (PDF) UC Berkeley/ University of Hohenheim; 2000.

34. Thompson JD, Higgins DG, Gibson TJ, CLUSTAL W: Improving the sensitivity of progressive multiple sequence alignment through sequence weighting, position-specific gap penalties and weight matrix choice. Nucleic Acids Res 1994, 22:4673-4680.
35. Felsenstein J: PHYLIP-Phylogeny Inference Package (Version 3.2). Cladistics 1989, 5:164-166.

36. Page RDM: TreeView: an application to display phylogenetic trees on personal computers. Comput Appl Biosci 1996, 12:357-358.

37. Hall TA: BioEdit: a user-friendly biological sequence alignment editor and analysis program for Windows 95/98/NT. Nucleic Acids Symp Ser 1999, 41:95-98.

38. Bradley DE: Ultrastructure of bacteriophages and bacteriocins. Bacteriol Rev 1967, 31(4):230-314.

doi:10.1186/1471-2164-13-224

Cite this article as: Lin and Lin: Genome-wide characterization of vibrio phage $\varphi$ pp2 with unique arrangements of the mob-like genes. $B M C$ Genomics 2012 13:224.

\section{Submit your next manuscript to BioMed Central and take full advantage of:}

- Convenient online submission

- Thorough peer review

- No space constraints or color figure charges

- Immediate publication on acceptance

- Inclusion in PubMed, CAS, Scopus and Google Scholar

- Research which is freely available for redistribution

Submit your manuscript at www.biomedcentral.com/submit
C Biomed Central 